\title{
LOWER SEMICONTINUITY OF MULTIPLE $\mu$-QUASICONVEX INTEGRALS
}

\author{
ILARIA FrAGALÀ ${ }^{1}$
}

\begin{abstract}
Lower semicontinuity results are obtained for multiple integrals of the kind $\int_{\mathbb{R} n} f\left(x, \nabla_{\mu} u\right) \mathrm{d} \mu$, where $\mu$ is a given positive measure on $\mathbb{R}^{n}$, and the vector-valued function $u$ belongs to the Sobolev space $H_{\mu}^{1, p}\left(\mathbb{R}^{n}, \mathbb{R}^{m}\right)$ associated with $\mu$. The proofs are essentially based on blow-up techniques, and a significant role is played therein by the concepts of tangent space and of tangent measures to $\mu$. More precisely, for fully general $\mu$, a notion of quasiconvexity for $f$ along the tangent bundle to $\mu$, turns out to be necessary for lower semicontinuity; the sufficiency of such condition is also shown, when $\mu$ belongs to a suitable class of rectifiable measures.
\end{abstract}

Mathematics Subject Classification. 28A25, 49J45, 26B25.

Received February 25, 2002.

\section{INTRODUCTION}

A large area in the Calculus of Variations deals with the lower semicontinuity of integral functionals whose integrand depends on the gradient of some function $u$. The interest in this feature arises from the so-called direct method [14]. It provides the existence of some minima for the involved functional as soon as it satisfies the assumption of being both lower semicontinuous and coercive with respect to some topology on the space of admissible functions $u$.

If we focus attention on a functional $J$ of the kind

$$
J(u)=\int_{\Omega} j(x, \nabla u) \mathrm{d} x,
$$

where $\Omega$ is a bounded open set in $\mathbb{R}^{n}$, and $j$ is a Carathéodory integrand satisfying standard $p$-growth conditions, the natural definition domain of $J$ is the classic Sobolev space $W^{1, p}(\Omega)$. While in the scalar case the crucial condition for the weak lower semicontinuity of $J$ on $W^{1, p}(\Omega)$ is the convexity of $j$ in its second variable [21,29], it is well-known that, in the elasticity case when $u$ is vector-valued, the appropriate assumption which replaces the usual convexity is the notion of quasiconvexity. It was first introduced by Morrey [28], and many relevant generalizations and refinements have followed. A bibliography, for sure not complete, can be found in $[1,3-5$, $12,14,17,22,23,25,26]$, and references therein.

Recently, integral functionals of the form (1.1), where the Lebesgue measure $\mathcal{L}^{n}$ over $\Omega$ is replaced by a general positive and finite Borel measure $\mu$ on $\mathbb{R}^{n}$, have been considered in order to model the elastic energy

Keywords and phrases: Borel measures, tangent properties, lower semicontinuity.

1 Dipartimento di Matematica, Politecnico di Milano, Piazza Leonardo da Vinci 32, 20133 Milano, Italy;

e-mail: fragala@mate.polimi.it 
of low-dimensional structures such as threads, membranes, or multijunction bodies [9]. In this framework, the integral energy in $\mathrm{d} \mu$ is a priori defined only for smooth displacements $u$, so that it seems natural to perform its relaxation [13] with respect to the $L_{\mu}^{p}$-topology. Under convexity assumption on $j$, this leads to a new integral functional in $\mathrm{d} \mu$, of the form $\bar{J}(u)=\int_{\mathbb{R}^{n}} j_{\mu}\left(x, \nabla_{\mu} u\right) \mathrm{d} \mu$, where $j_{\mu}$ is related to $j$ by an inf-convolution formula (so it is still a convex integrand), $\nabla_{\mu} u$ is the $\mu$-tangential gradient of $u$, and the definition domain of $\bar{J}$ is a suitable notion of Sobolev space $H_{\mu}^{1, p}\left(\mathbb{R}^{n}, \mathbb{R}^{m}\right)$ (possibly being $m>1$ ) [9].

One is thus led to a framework which draws an exact parallel with the Lebesgue usual setting, and of course the choice of taking $\mu$ equal to some Hausdorff measure $\mathcal{H}^{k}$ on a $k$-dimensional subset of $\mathbb{R}^{n}$ is of special interest. In particular, if in the vectorial case $m>1$ one starts from an integral functional of the kind

$$
F(u)=\int_{\mathbb{R}^{n}} f\left(x, \nabla_{\mu} u\right) \mathrm{d} \mu, \quad u \in H_{\mu}^{1, p}\left(\mathbb{R}^{n}, \mathbb{R}^{m}\right),
$$

it is natural to ask whether some condition on $f$, corresponding to the usual quasiconvexity, can be shown to be necessary and sufficient for the weak lower semicontinuity of $F$. The aim of this paper is to give an answer to such question. We are also motivated by the fact that, in many variational problems involving low-dimensional structures in $\mathbb{R}^{n}$, identifying them to measures and working in the framework just sketched above, seems to be a very fruitful approach (we refer to $[6,8,10,11]$ for applications in the fields of shape optimization, optimal control, and homogenization, see also [34]).

A central role in the development of the tangential calculus with respect to $\mu$ is played by the notion of tangent bundle $T_{\mu}$, which has been introduced in [9], and subsequently studied in [18]. The main result of the present paper states roughly that, when $\mu$ is concentrated over a rectifiable set, the functional defined in (1.2) is weakly lower semicontinuous if and only if the integrand $f(x, \cdot)$ is quasiconvex along the directions of $T_{\mu}(x)$ for $\mu$-a.e. $x$ (cf. Def. 3.1).

In fact, the necessity of such condition can be proved for a general measure $\mu$, by a contradiction argument based on the Vitali covering theorem (see Th. 4.1); we also heavily exploit the notion of tangent measures to $\mu$ introduced by Preiss in [31], and its connection with $T_{\mu}$ established in [18].

The sufficiency is much more delicate, and it is proved only for a class of rectifiable measures, though we do not have a counter-example showing that such class cannot be enlarged. As a key tool, we make use of a rather involved notion of $\mu$-quasiconvexity by blow-up for the integrand $f$ (cf. Def. 3.4). In fact, if $\mu$ is a doubling measure satisfying a $p$-Poincaré inequality, like the one investigated in [19], we can prove that the $\mu$-quasiconvexity by blow-up is necessary and sufficient for lower semicontinuity (see Ths. 4.2 and 5.1). In particular, sufficiency is proved adopting the blow-up technique of Fonseca and Müller. So far, for a general measure $\mu$, we can prove only that the $\mu$-quasiconvexity by blow-up entails the $T_{\mu}$-quasiconvexity (see Prop. 3.5). The converse implication is also true, under the assumption that $\mu$ is a rectifiable varifold with bounded first variation (see Prop. 3.6). Thus, whenever such a $\mu$ satisfies a $p$-Poincaré inequality, the $T_{\mu}$-quasiconvexity turns out to be a sharp condition for semicontinuity.

Under the suitable growth conditions on $f$, our results can be summed up through the following scheme, where each implication needs the assumptions on $\mu$ placed at the right hand side of the corresponding arrow: in particular, simple arrow means full generality, whereas letters (P), (D) and (R) denote respectively the Poincaré inequality, the doubling condition, and the rectifiability property with bounded first variation. $F$ weakly lower semicontinuous on $H_{\mu}^{1, p}$

$$
\begin{array}{ccc}
\Downarrow(\mathrm{P}) \Uparrow(\mathrm{P})-(\mathrm{D}) & \Downarrow \Uparrow(\mathrm{P})-(\mathrm{R}) \\
f \text { convex } \Rightarrow(\mathrm{P}) f \mu \text {-quasiconvex by blow-up } & \Rightarrow f T_{\mu} \text { quasiconvex } \\
& \Leftarrow(\mathrm{R})
\end{array}
$$

The paper is organized as follows:

Section 2 is a concise summary of the basic notation, definitions, and preliminary results. 
In Section 3, we introduce different concepts of quasiconvexity with respect to $\mu$, and we establish some comparison results among them.

The statements and proofs of the main theorems, respectively for what concerns necessary and sufficient conditions for the lower semicontinuity of the functional introduced in (1.2), are contained in Sections 4 and 5 .

\section{Notation AND PRELIMINARIES}

Throughout the paper, we let $\mu$ be a positive Radon measure, compactly supported in $\mathbb{R}^{n}$, and we omit the integration domain whenever it coincides with the whole $\mathbb{R}^{n}$.

In order to give a rigorous interpretation to the functional $F$ defined by (1.2), we need the definitions of Sobolev functions with respect to $\mu$, and of their $\mu$-tangential gradient.

We begin by recalling the concept of tangent bundle to $\mu$ introduced in [9]; at $\mu$-a.e. $x \in \mathbb{R}^{n}$, it is given by

$$
T_{\mu}(x):=\mu-\operatorname{ess} \bigcup\left\{\Phi(x): \Phi \in L_{\mu}^{1}\left(\mathbb{R}^{n}, \mathbb{R}^{n}\right), \operatorname{div}(\Phi \mu) \in \mathcal{M}\right\}
$$

where the divergence operator is intended in the distributional sense, and $\mathcal{M}$ denotes the set of signed Borel measures with finite total variation on $\mathbb{R}^{n}$. By the definition of $\mu$-essential union, $T_{\mu}(x)$ is the multifunction characterized by the following two properties (see [33]):

(i) for all $\Phi \in L_{\mu}^{1}\left(\mathbb{R}^{n}, \mathbb{R}^{n}\right)$ with $\operatorname{div}(\Phi \mu) \in \mathcal{M}$, the inclusion $\Phi(x) \in T_{\mu}(x)$ holds $\mu$-a.e.;

(ii) if $\Sigma$ is any other $\mu$-measurable multifunction, from $\mathbb{R}^{n}$ into the closed sets of $\mathbb{R}^{n}$, such that the inclusion $\Phi(x) \in \Sigma(x)$ holds $\mu$-a.e. for all fields $\Phi \in L_{\mu}^{1}\left(\mathbb{R}^{n}, \mathbb{R}^{n}\right)$ with $\operatorname{div}(\Phi \mu) \in \mathcal{M}$, then we have $T_{\mu}(x) \subseteq \Sigma(x)$ $\mu$-a.e.

One can check that, for $\mu$-a.e. $x \in \mathbb{R}^{n}, T_{\mu}(x)$ individuates a linear subspace of $\mathbb{R}^{n}$, whose dimension may depend on $x$; further, when $\mu$ is the Hausdorff measure $\mathcal{H}^{k}$ over a $k$-Lipschitz manifold $S, T_{\mu}$ coincides $\mu$-a.e. with the usual tangent space to $S$.

Throughout the paper, we shall consider measures $\mu$ such that $T_{\mu}(x)$ is nonzero for $\mu$-a.e. $x$. Notice in particular that Dirac masses are ruled out of our framework.

For the reader's ease, we recall below a comparison result, often used in the following, which relates definition (2.1) to the notion of tangent measures to $\mu$ introduced by Preiss in [31]. Unless otherwise specified, $B$ will denote the unit ball of $\mathbb{R}^{n}$. A measure $\nu$ on $B$ is called a tangent measure to $\mu$ at $x$, if there exists a sequence of positive real numbers $\rho_{i}$, converging to zero, such that the blown-up measures $\frac{\mu_{x, \rho_{i}}}{\mu\left(B_{\rho_{i}}(x)\right)}$ converge weakly $*$ to $\nu$ on $B$ as $i \rightarrow+\infty$, where $\mu_{x, \rho_{i}}(B):=\mu\left(x+\rho_{i} B\right)$, and $B_{\rho_{i}}(x)$ denotes the ball of radius $\rho_{i}$ centered at $x$ (see also Chap. 14 of [27]). Letting $\operatorname{Tan}(\mu, x)$ be the class of all tangent measures to $\mu$ at $x$, the following product decomposition holds, whose proof can be found in [18] (Lem. 2.6).

Lemma 2.1. For $\mu$-a.e. $x \in \mathbb{R}^{n}$, any measure $\nu \in \operatorname{Tan}(\mu, x)$ is of the form

$$
\left(\mathcal{H}_{T_{\mu}(x)}^{k(x)} \times \sigma\right)\llcorner B
$$

where $k(x)$ is the dimension of $T_{\mu}(x), \mathcal{H}_{T_{\mu}(x)}^{k(x)}$ is the $k(x)$-dimensional Hausdorff measure over $T_{\mu}(x)$, and $\sigma$ is a measure on the orthogonal complement of $T_{\mu}(x)$.

For general $\mu$, the set $\operatorname{Tan}(\mu, x)$ can be very large [30]. This is not the case when $\mu$ is a measure that in the sequel will be called $k$-rectifiable, namely of the kind $\theta \mathcal{H}^{k} L S$, with $k$ an integer, $S$ a countably $k$-rectifiable subset of $\mathbb{R}^{n}$, and $\theta$ a positive function $\mathcal{H}^{k}$-integrable on $S$ [16] (3.2.14). Using the varifolds' language, $\mu$ is the weight measure of the rectifiable varifold $V=(S, \theta)$ [32] (Sect. 15). For such a measure $\mu$, at $\mu$-a.e. $x$ there exists a $k$-dimensional subspace $T_{S}(x)$ of $\mathbb{R}^{n}$, which is said to be the approximate tangent space to $\mu$ at $x$, such that, when $\rho$ tends to zero, the measures $\frac{1}{\rho^{k}} \mu_{x, \rho}$ converge weakly star to $\theta(x) \mathcal{H}^{k}\left\llcorner T_{S}(x)[32]\right.$ (Th. 11.6). 
It can be proved that in general $T_{\mu}$ is contained $\mu$-a.e. into $T_{S}$, see [9] (Lem. 5.2) or [18] (Lem. 2.4). Moreover, we recall below a result proved in [18] (Th. 3.5), stating that $T_{\mu}$ agrees with the approximate tangent space to $\mu$ for rectifiable measures $\mu$ which are regular enough in terms of their first variation [32] (Sect. 39). We notice that such measures are closely related to those with bounded curvature introduced in [7] (Sect. 2).

Lemma 2.2. Let $\mu=\theta \mathcal{H}^{k}\llcorner S$ be the weight measure of a $k$-rectifiable varifold $V=(S, \theta)$, and suppose that the first variation of $V$ is a Radon measure. Then $T_{\mu}$ coincides $\mu$-a.e. with the approximate tangent space $T_{S}$.

We turn now attention to the Sobolev space $H_{\mu}^{1, p}\left(\mathbb{R}^{n}, \mathbb{R}^{m}\right)$. We let $p$ belong to $(1,+\infty)$, and $\|\cdot\|_{p, \mu}$ be the usual $L_{\mu}^{p}$-norm. Further, we denote by $P_{\mu}(x)$ the orthogonal projector onto $T_{\mu}(x)$. The space $H_{\mu}^{1, p}\left(\mathbb{R}^{n}, \mathbb{R}^{m}\right)$ is by definition the completion of $\mathcal{D}\left(\mathbb{R}^{n}, \mathbb{R}^{m}\right)$ with respect to the norm $\|\psi\|_{1, p, \mu}:=\|\psi\|_{p, \mu}+\left\|\nabla_{\mu} \psi\right\|_{p, \mu}$, where $\mathcal{D}:=\mathcal{C}_{0}^{\infty}$, and $\nabla_{\mu} \psi(x)$ is the $P_{\mu}(x)$-projection (by components) of $\nabla \psi(x)$. (For the sake of precision, we have to remark that $\|\cdot\|_{1, p, \mu}$ is rather a seminorm than a norm on the space $\mathcal{D}$; however, it becomes a norm on the quotient space of $\mathcal{D}$ with respect to equality $\mu$-almost everywhere.)

An equivalent way to define the space $H_{\mu}^{1, p}\left(\mathbb{R}^{n}, \mathbb{R}^{m}\right)$ is saying that the linear operator $A: D(A)$ $\subset L_{\mu}^{p}\left(\mathbb{R}^{n}, \mathbb{R}^{m}\right) \rightarrow L_{\mu}^{p}\left(\mathbb{R}^{n}, \mathbb{R}^{n m}\right)$ defined by $D(A)=\mathcal{D}\left(\mathbb{R}^{n}, \mathbb{R}^{m}\right)$ and $A \psi=\nabla_{\mu} \psi$, is closable [9] (Prop. 2.1), and the domain of its unique closed extension $\bar{A}$ is called $H_{\mu}^{1, p}\left(\mathbb{R}^{n}, \mathbb{R}^{m}\right)$. So, for any $u \in H_{\mu}^{1, p}\left(\mathbb{R}^{n}, \mathbb{R}^{m}\right)$, it results defined by completion a $\mu$-tangential gradient $\nabla_{\mu} u \in L_{\mu}^{p}\left(\mathbb{R}^{n}, \mathbb{R}^{n m}\right)$. We also stress that $H_{\mu}^{1, p}\left(\mathbb{R}^{n}, \mathbb{R}^{m}\right)$ is a reflexive Banach space endowed with the norm $\|u\|_{1, p, \mu}:=\|u\|_{p, \mu}+\left\|\nabla_{\mu} u\right\|_{p, \mu}$. In such space, the weak convergence is characterized by

$$
u \rightarrow u \text { weakly in } H_{\mu}^{1, p}\left(\mathbb{R}^{n}, \mathbb{R}^{m}\right) \Longleftrightarrow \begin{cases}u_{h} \rightarrow u & \text { weakly in } L_{\mu}^{p}\left(\mathbb{R}^{n}, \mathbb{R}^{m}\right), \\ \nabla_{\mu} u_{h} \rightarrow \nabla_{\mu} u & \text { weakly in } L_{\mu}^{p}\left(\mathbb{R}^{n}, \mathbb{R}^{m n}\right) .\end{cases}
$$

We can now give a more precise statement of the relaxation result mentioned in the Introduction. We specify it to the case $j(z)=|z|^{p}$, namely we consider the functional

$$
J(u):= \begin{cases}\int_{\mathbb{R}^{n}}|\nabla u|^{p} \mathrm{~d} \mu & \text { if } u \in \mathcal{D}\left(\mathbb{R}^{n}, \mathbb{R}^{m}\right) \\ +\infty & \text { otherwise }\end{cases}
$$

We recall that the relaxed functional $\bar{J}$ of $J$ with respect to the $L_{\mu}^{p}\left(\mathbb{R}^{n}, \mathbb{R}^{m}\right)$-topology, is obtained by definition as

$$
\bar{J}(u)=\inf \left\{\liminf _{h \rightarrow+\infty} J\left(u_{h}\right): u_{h} \rightarrow u \text { in } L_{\mu}^{p}\left(\mathbb{R}^{n}, \mathbb{R}^{m}\right)\right\}
$$

Moreover, respectively due to the convexity of $J$ and to the reflexivity of $H_{\mu}^{1, p}\left(\mathbb{R}^{n}, \mathbb{R}^{m}\right), \bar{J}$ coincides with the relaxed functional with respect to the weak topology on $L_{\mu}^{p}\left(\mathbb{R}^{n}, \mathbb{R}^{m}\right)$ and on $H_{\mu}^{1, p}\left(\mathbb{R}^{n}, \mathbb{R}^{m}\right)$. The explicit expression of $\bar{J}$ is given by [9] (Th. 3.1):

$$
\bar{J}(u)= \begin{cases}\int_{\mathbb{R}^{n}}\left|\nabla_{\mu} u\right|^{p} \mathrm{~d} \mu & \text { if } u \in H_{\mu}^{1, p}\left(\mathbb{R}^{n}, \mathbb{R}^{m}\right) \\ +\infty & \text { otherwise. }\end{cases}
$$

We also point out that the relaxation of nonconvex integral functionals in $\mathrm{d} \mu$, when $\mu$ is concentrated over a smooth manifold, has been recently studied in [24]. 


\section{3. $\mu$-QUASICONVEXITY}

We begin by formulating the notion of quasiconvexity on a $k$-dimensional subspace $\pi$ of $\mathbb{R}^{n}$. Let $\mathrm{g}$ be a Borel measurable and locally integrable real function on $\mathcal{L}\left(\pi, \mathbb{R}^{m}\right)$, the class of linear mappings from $\pi$ to $\mathbb{R}^{m}$, and set $\omega_{k}:=\mathcal{H}^{k}(B \cap \pi)$.

Definition 3.1. We say that $g$ is $\pi$-quasiconvex if

$$
g(A) \leq \frac{1}{\omega_{k}} \int_{B \cap \pi} g(A+\nabla \psi(y)) \mathrm{d} \mathcal{H}^{k}(y)
$$

for every $A \in \mathcal{L}\left(\pi, \mathbb{R}^{m}\right)$ and for every $\psi \in \mathcal{D}\left(B \cap \pi, \mathbb{R}^{m}\right)$.

Remark 3.2. As in the usual case $\pi=\mathbb{R}^{n}$, one can easily verify that, if $m=1$ or $k=1$, the $\pi$-quasiconvexity is equivalent to the convexity on $\pi$; moreover, if we assume (3.1), the same inequality holds for any test function $\psi \in W_{0}^{1, \infty}\left(\Omega \cap \pi, \mathbb{R}^{m}\right)$ whatever domain $\Omega \subseteq \mathbb{R}^{n}$, i.e.

$$
g(A) \leq \frac{1}{\mathcal{H}^{k}(\Omega \cap \pi)} \int_{\Omega \cap \pi} g(A+\nabla \psi(y)) \mathrm{d} \mathcal{H}^{k}(y)
$$

for every $A \in \mathcal{L}\left(\pi, \mathbb{R}^{m}\right)$ and for every $\psi \in W_{0}^{1, \infty}\left(\Omega \cap \pi, \mathbb{R}^{m}\right)(c f .[14], 4.1)$.

With regard to the functional $F$ defined in (1.2), we have in mind to apply Definition 3.1 to the case $g=f(x, \cdot)$ and $\pi=T_{\mu}(x)$; at this aim, the next result will be useful in connection with Lemma 2.1.

Proposition 3.3. The following conditions are equivalent:

(i) $g$ is $\pi$-quasiconvex;

(ii) $g$ satisfies the inequality

$$
g(A) \leq \frac{1}{\omega_{k}} \int_{B \cap \pi} g\left(A+\nabla_{\pi} \varphi(y)\right) \mathrm{d} \mathcal{H}^{k}(y)
$$

for every $A \in \mathcal{L}\left(\pi, \mathbb{R}^{m}\right)$ and for every $\varphi \in \mathcal{D}\left(B, \mathbb{R}^{m}\right)$, where $\nabla_{\pi} \varphi$ denotes the projection by components of the Jacobian matrix of $\varphi$ on $\pi$;

(iii) for any measure $\nu$ of the kind $\left(\mathcal{H}^{k}\llcorner\pi) \times \sigma\right.$, with $\sigma$ a nonzero measure on $\pi^{\perp}$, it holds

$$
g(A) \leq \frac{1}{\nu(B)} \int_{B} g\left(A+\nabla_{\pi} \varphi(y, z)\right) \mathrm{d} \nu(y, z)
$$

for every $A \in \mathcal{L}\left(\pi, \mathbb{R}^{m}\right)$ and for every $\varphi \in \mathcal{D}\left(B, \mathbb{R}^{m}\right)$;

(iv) there exists a measure $\nu$ of the kind $\left(\mathcal{H}^{k}\llcorner\pi) \times \sigma\right.$, with $\sigma$ a nonzero measure on $\pi^{\perp}$, such that (3.2) holds for every $A \in \mathcal{L}\left(\pi, \mathbb{R}^{m}\right)$ and for every $\varphi \in \mathcal{D}\left(B, \mathbb{R}^{m}\right)$.

Proof. $(i) \Rightarrow($ ii $)$. For every $\varphi \in \mathcal{D}\left(B, \mathbb{R}^{m}\right)$, let $\varphi_{\left.\right|_{\pi}}$ be the restriction of $\varphi$ on $B \cap \pi$. Then $\varphi_{\left.\right|_{\pi}}$ belongs to $\mathcal{D}\left(B \cap \pi, \mathbb{R}^{m}\right)$, and for every $y \in B \cap \pi$ we have $\nabla_{\pi} \varphi(y)=\nabla \varphi_{\left.\right|_{\pi}}(y)$. So it is enough to take $\psi=\varphi_{\left.\right|_{\pi}}$ in the definition of $\pi$-quasiconvexity.

$(i i) \Rightarrow(i)$. Let $\psi \in \mathcal{D}\left(\pi, \mathbb{R}^{m}\right)$ and for $(y, z) \in \mathbb{R}^{n}=\pi \times \pi^{\perp}$ let us denote $\bar{\psi}(y, z)$ the function, not depending on $z$, given by $\psi(y)$. Then $\bar{\psi}$ is regular on $\mathbb{R}^{n}$, but it has not a compact support, so it cannot be taken as a test 
function in (ii). Therefore we consider a cut-off function depending only on the orthogonal component $z$, that is a smooth function $\eta$ on $\pi^{\perp}$ such that

$$
\begin{cases}\eta(z)=0 & \text { for } z \in \pi^{\perp},\|z\| \geq 1 \\ \eta(z)=1 & \text { for } z \in \pi^{\perp},\|z\| \leq \frac{1}{2}\end{cases}
$$

Now if we set $\varphi(y, z)=\psi(y) \eta(z), \varphi$ has compact support, and we have

$$
\nabla \varphi(y, z)=(\nabla \psi(y) \eta(z), \psi(y) \nabla \eta(z)),
$$

so that, for every $y \in(B \cap \pi)$,

$$
\nabla_{\pi} \varphi(y)=\nabla \psi(y) \eta(0)=\nabla \psi(y)
$$

Thus, taking $\varphi$ as a test function in (ii), we get that (3.1) is satisfied for the corresponding $\psi$.

(ii) $\Rightarrow($ iii $)$. Let $A \in \mathcal{L}\left(\pi, \mathbb{R}^{m}\right)$, and $\varphi \in \mathcal{D}\left(B, \mathbb{R}^{m}\right)$. Let $Q=L \times M$ be a cube containing $B$, with $L$ and $M$ parallel respectively to $\pi$ and $\pi^{\perp}$. Since, for any fixed $z \in M$, the function $y \mapsto \varphi(y, z)$ belongs to $\mathcal{D}\left(L, \mathbb{R}^{m}\right)$, we have

$$
\mathcal{H}^{k}(L) g(A) \leq \int_{L} g\left(A+\nabla_{\pi} \varphi(y, z)\right) \mathrm{d} \mathcal{H}^{k}(y)
$$

Integrating the above inequality on $M$ in $\mathrm{d} \sigma(z)$, applying Fubini's theorem, and recalling that $\varphi$ is supported on $B$, we obtain

$$
\begin{aligned}
\nu(Q) g(A) & \leq \int_{M} \int_{L} g\left(A+\nabla_{\pi} \varphi(y, z)\right) \mathrm{d} \mathcal{H}^{k}(y) \mathrm{d} \sigma(z) \\
& =\int_{Q} g\left(A+\nabla_{\pi} \varphi(y, z)\right) \mathrm{d} \nu(y, z) \\
& =\int_{B} g\left(A+\nabla_{\pi} \varphi(y, z)\right) \mathrm{d} \nu(y, z)+\nu(Q \backslash B) g(A),
\end{aligned}
$$

whence (3.2).

$($ iii $) \Rightarrow($ ii). It is enough to take the measure $\sigma$ equal to a Dirac mass concentrated at zero.

$(i i i) \Rightarrow(i v)$. It is immediate.

(iv) $\Rightarrow(i i)$. Let $Q=L \times M$ be a cube contained in $B$, with $L$ and $M$ parallel respectively to $\pi$ and $\pi^{\perp}$. Since

(iv) holds by hypothesis, we deduce

$$
g(A) \leq \frac{1}{\mathcal{H}^{k}(L) \sigma(M)} \int_{L} \int_{M} g\left(A+\nabla_{\pi} \varphi(y, z)\right) \mathrm{d} \mathcal{H}^{k}(y) \mathrm{d} \sigma(z)
$$

for every $A \in \mathcal{L}\left(\pi, \mathbb{R}^{m}\right)$ and for every $\varphi \in \mathcal{D}\left(L \times M, \mathbb{R}^{m}\right)$.

Arguing as in the proof of the implication $(i i) \Rightarrow(i)$, that is using a cut-off function depending only on the coordinates orthogonal to $\pi$, we see that the above inequality holds for a larger class of test functions; we mean that it remains true when $\varphi$ is a regular function supported in $L^{\prime} \times \mathbb{R}^{n-k}$, with $L^{\prime} \subset \subset L$. Then for every $\varphi \in \mathcal{D}\left(L \times M, \mathbb{R}^{m}\right)$ and for every $\epsilon>0$, if we set $\varphi_{\epsilon}(y, z)=\varphi(y, \epsilon z), \varphi_{\epsilon}$ is still admissible as a test function. Hence we have

$$
g(A) \leq \frac{1}{\mathcal{H}^{k}(L) \sigma(M)} \int_{L} \int_{M} g\left(A+\nabla_{\pi} \varphi(y, \epsilon z)\right) \mathrm{d} \mathcal{H}^{k}(y) \mathrm{d} \sigma(z) .
$$


This implies the existence of a point $z_{\epsilon} \in M$ such that

$$
g(A) \leq \frac{1}{\mathcal{H}^{k}(L)} \int_{L} g\left(A+\nabla_{\pi} \varphi\left(y, \epsilon z_{\epsilon}\right)\right) \mathrm{d} \mathcal{H}^{k}(y)
$$

Finally, passing to the limit for $\epsilon$ tending to zero, we get

$$
g(A) \leq \frac{1}{\mathcal{H}^{k}(L)} \int_{L} g\left(A+\nabla_{\pi} \varphi(y, 0)\right) \mathrm{d} \mathcal{H}^{k}(y)
$$

We turn now attention to the case $\pi=T_{\mu}(x)$, and $g=f(x, \cdot)$. In general, the condition that $f(x, \cdot)$ is quasiconvex in the classical sense does not ensure that $f(x, \cdot)$ is $T_{\mu}(x)$-quasiconvex. However, examples of functions $f$ such that $f(x, \cdot)$ is $T_{\mu}(x)$-quasiconvex for $\mu$-a.e. $x$ can be produced using Proposition 3.3. Indeed, a sufficient condition for $T_{\mu}$-quasiconvexity, which can be concretely handled, is the quasiconvexity of $f\left(x, P_{\mu}(x) \cdot\right)$. For instance, consider the measure $\mu$ defined on $\mathbb{R}^{3}$ by

$$
\mu=\mu_{1}+\mu_{2}=\mathcal{H}^{1}\left\llcorner\left(B \cap\left\{x_{1}=x_{2}=0\right\}\right)+\mathcal{H}^{2}\left\llcorner\left(B \cap\left\{x_{3}=0\right\}\right),\right.\right.
$$

and let $f(x, \cdot)$ be given on $\mathcal{L}\left(\mathbb{R}^{3}, \mathbb{R}^{3}\right)$ by

$$
f(x, A)= \begin{cases}A_{31}^{2}+A_{32}^{2}+A_{33}^{2} & \text { for } \mu_{1} \text {-a.e. } x \\ \left(A_{11} A_{22}-A_{12} A_{21}\right)^{2}+\left(A_{12} A_{23}-A_{13} A_{22}\right)^{2} & \text { for } \mu_{2}-\text { a.e. } x\end{cases}
$$

Since $f\left(x, P_{\mu}(x) \cdot\right)$ is polyconvex, it is quasiconvex in the usual sense on $\mathcal{L}\left(\mathbb{R}^{3}, \mathbb{R}^{3}\right)$; then, by applying the implication $(i v) \Rightarrow(i)$ of Proposition 3.3, we deduce that $f(x, \cdot)$ is $T_{\mu}(x)$-quasiconvex. We now introduce a notion of $\mu$-quasiconvexity by blow up. We assume throughout the remaining of the section that $f$ is a Carathéodory integrand satisfying, for a fixed exponent $p \in(1,+\infty)$, and some constant $C>0$, a growth condition of the kind $f(x, z) \leq C\left(1+|z|^{p}\right)$. For any fixed $x \in \mathbb{R}^{n}$, we consider the class of admissible radius (cf. [5] Def. 2.1),

$$
\mathcal{R}_{x}:=\left\{\rho>0: \mu\left(\partial B_{\rho}(x)\right)=0\right\}
$$

and the class of families admissible by blow-up at $x$, defined by

$$
\mathcal{F}_{x}:=\left\{\left\{\varphi_{\rho}\right\}_{\rho \in \mathcal{R}_{x}} \subset \mathcal{D}\left(B, \mathbb{R}^{m}\right): \sup _{\rho \in \mathcal{R}_{x}} \frac{1}{\mu\left(B_{\rho}(x)\right)} \int_{B}\left|\nabla \varphi_{\rho}\right|^{p} \mathrm{~d} \mu^{x, \rho}<+\infty\right\}
$$

Definition 3.4. We say that $f(x, \cdot)$ is $\mu$-quasiconvex by blow-up if the inequality

$$
f(x, A) \leq \liminf _{\mathcal{R}_{x} \ni \rho \rightarrow 0^{+}} \frac{1}{\mu\left(B_{\rho}(x)\right)} \int_{B} f\left(x, A+P_{\mu}(x) \nabla \varphi_{\rho}\right) \mathrm{d} \mu^{x, \rho}
$$

holds for any $A \in \mathcal{L}\left(T_{\mu}(x), \mathbb{R}^{m}\right)$ and any admissible family $\left\{\varphi_{\rho}\right\} \subset \mathcal{F}_{x}$ (where $P_{\mu}(x)$ is the orthogonal projector onto $\left.T_{\mu}(x)\right)$.

Inequality (3.3) will be shown to be the key for the lower semicontinuity of the functional $F$ in (1.2). Therefore, it is interesting to compare it with the $T_{\mu}(x)$-quasiconvexity of $f(x, \cdot)$ according to Definition 3.1. We are going to prove that, for any measure $\mu$, the $\mu$-quasiconvexity by blow-up implies the $T_{\mu}$-quasiconvexity; moreover the two notions agree for suitable rectifiable measures. Establishing whether this is true also for general $\mu$ remains by now a delicate open problem (see also Cor. 4.3). 
Proposition 3.5. If $f(x, \cdot)$ is $\mu$-quasiconvex by blow-up for $\mu$-a.e. $x$, then it is $T_{\mu}(x)$-quasiconvex for $\mu$-a.e. $x$.

Proof. It is not restrictive to deal with a fixed point $x$ where the tangent space $T_{\mu}(x)$ is well-defined, and where any tangent measure to $\mu$ satisfies the product decomposition of Lemma 2.1. Let us show that, if (3.3) holds, then condition $(i v)$ of Proposition 3.3 is satisfied for some element $\nu_{x} \in \operatorname{Tan}(\mu, x)$. Indeed, let $A \in \mathcal{L}\left(T_{\mu}(x), \mathbb{R}^{m}\right)$ and $\varphi \in \mathcal{D}\left(B, \mathbb{R}^{m}\right)$. We notice that $\varphi$ may be taken as an admissible family (independent of $\rho$ ) in the class $\mathcal{F}_{x}$. Thus, let us select a sequence of radius $\left\{\rho_{i}\right\}$ tending to zero which achieves the liminf at the right hand side of (3.3) (with $\varphi_{\rho}=\varphi$ ), and such that $\frac{\mu^{x, \rho_{i}}}{\mu\left(B_{\rho_{i}}(x)\right)}$ converge weakly star to a tangent measure $\nu_{x}$ over $B$. We stress that $\nu_{x}$ is nonzero, because $\frac{\mu^{x, \rho_{i}}}{\mu\left(B_{\rho_{i}}(x)\right)}(B)=1$ for every $i$. We obtain

$$
\begin{aligned}
f(x, A) & \leq \liminf _{\mathcal{R}_{x} \ni \rho \rightarrow 0^{+}} \frac{1}{\mu\left(B_{\rho}(x)\right)} \int_{B} f\left(x, A+P_{\mu}(x) \nabla \varphi\right) \mathrm{d} \mu^{x, \rho}=\lim _{i \rightarrow \infty} \frac{1}{\mu\left(B_{\rho_{i}}(x)\right)} \int_{B} f\left(x, A+P_{\mu}(x) \nabla \varphi\right) \mathrm{d} \mu^{x, \rho_{i}} \\
& =\int_{B} f\left(x, A+P_{\mu}(x) \nabla \varphi\right) \mathrm{d} \nu_{x} \leq \frac{1}{\nu_{x}(B)} \int_{B} f\left(x, A+P_{\mu}(x) \nabla \varphi\right) \mathrm{d} \nu_{x} .
\end{aligned}
$$

Proposition 3.6. Let $\mu$ be the weight measure of a $k$-rectifiable varifold with bounded first variation. If $f(x, \cdot)$ is Lipschitz and $T_{\mu}(x)$-quasiconvex for $\mu$-a.e. $x$, then it is $\mu$-quasiconvex by blow-up for $\mu$-a.e. $x$.

Proof. Let $\mu=\theta \mathcal{H}^{k}\left\llcorner S\right.$. In view of Lemma 2.2, it is not restrictive to select a point $x \in S$ such that $T_{\mu}(x)$ coincides with the approximate tangent space to $S$ at $x$. Moreover, we can choose $x$ as a Lebesgue point for the density function $\theta$.

Since $S$ is covered up to a $\mu$-negligible set by a union of $\mathcal{C}^{1}$ manifolds, we can assume that for $\rho$ sufficiently small the orthogonal projector $P_{\mu}(x): S \cap B_{\rho}(x) \rightarrow T_{\mu}(x)$ is a Lipschitz one-to-one map, whose Lipschitz constant tends to 1 as $\rho \rightarrow 0^{+}[2](3.15)$. We set $D_{\rho}(x):=P_{\mu}(x)\left[S \cap B_{\rho}(x)\right]$, and $g$ the inverse map $P_{\mu}(x)^{-1}$ defined on $D_{\rho}(x) ; g$ is also Lipschitz, with a constant tending to 1 as $\rho \rightarrow 0^{+}$.

Let $A \in \mathcal{L}\left(T_{\mu}(x), \mathbb{R}^{m}\right)$, and $\left\{\varphi_{\rho}\right\}_{\rho \in \mathcal{R}_{x}} \subset \mathcal{F}_{x}$. For each fixed $\rho \in \mathcal{R}_{x}$, setting $\varphi_{\rho}^{x, \rho}(y):=\rho \varphi_{\rho}\left(\frac{y-x}{\rho}\right)$, the $T_{\mu}(x)$-quasiconvexity of $f(x, \cdot)$ gives

$$
f(x, A) \leq \frac{1}{\mathcal{H}^{k}\left(D_{\rho}(x)\right)} \int_{D_{\rho}(x)} f\left(x, A+P_{\mu}(x) \nabla\left(\varphi_{\rho}^{x, \rho} \circ g\right)(y)\right) \mathrm{d} \mathcal{H}^{k}(y)
$$

We denote by $J P_{\mu}(x)$ the Jacobian of the map $P_{\mu}(x)$. Using the area formula [16] $(3.2 .22)$, since $\operatorname{Lip}\left(P_{\mu}(x)_{\left.\right|_{S \cap B}(x)}\right)$ $\rightarrow 1$ as $\rho \rightarrow 0^{+}$, we obtain

$$
\mathcal{H}^{k}\left(D_{\rho}(x)\right)=\int_{S \cap B_{\rho}(x)} J P_{\mu}(x) \mathrm{d} \mathcal{H}^{k}=\mathcal{H}^{k}\left(S \cap B_{\rho}(x)\right)+o\left(\mu\left(B_{\rho}(x)\right)\right)
$$


By the same argument, denoting by $\mathcal{O}(\rho)$ an infinitesimal with $\rho$, possibly varying from line to line, we have

$$
\begin{aligned}
& \int_{D_{\rho}(x)} f\left(x, A+P_{\mu}(x) \nabla\left(\varphi_{\rho}^{x, \rho} \circ g\right)(y)\right) \mathrm{d} \mathcal{H}^{k}(y) \\
= & \int_{D_{\rho}(x)} f\left(x, A+P_{\mu}(x) \nabla \varphi_{\rho}^{x, \rho}(g(y))(1+\mathcal{O}(\rho))\right) \mathrm{d} \mathcal{H}^{k}(y) \\
= & \int_{D_{\rho}(x)} f\left(x, A+P_{\mu}(x) \nabla \varphi_{\rho}^{x, \rho}(g(y))\right) \mathrm{d} \mathcal{H}^{k}(y)+o\left(\mu\left(B_{\rho}(x)\right)\right) \\
= & \int_{S \cap B_{\rho}(x)} f\left(x, A+P_{\mu}(x) \nabla \varphi_{\rho}^{x, \rho}(y)\right) J P_{\mu}(x) \mathrm{d} \mathcal{H}^{k}(y)+o\left(\mu\left(B_{\rho}(x)\right)\right) \\
= & \int_{S \cap B_{\rho}(x)} f\left(x, A+P_{\mu}(x) \nabla \varphi_{\rho}^{x, \rho}(y)\right)(1+\mathcal{O}(\rho)) \mathrm{d} \mathcal{H}^{k}(y)+o\left(\mu\left(B_{\rho}(x)\right)\right) \\
= & \int_{S \cap B_{\rho}(x)} f\left(x, A+P_{\mu}(x) \nabla \varphi_{\rho}^{x, \rho}(y)\right) \mathrm{d} \mathcal{H}^{k}(y)+o\left(\mu\left(B_{\rho}(x)\right)\right) \\
= & \frac{1}{\theta(x)} \int_{B} f\left(x, A+P_{\mu}(x) \nabla \varphi_{\rho}(y)\right) \mathrm{d} \mu^{x, \rho}(y)+o\left(\mu\left(B_{\rho}(x)\right)\right),
\end{aligned}
$$

where we used also the Lipschitz regularity of $f(x, \cdot)$, the assumption $\left\{\varphi_{\rho}\right\} \subset \mathcal{F}_{x}$ together with the $p$-growth of $f$, and the fact that $x$ is a Lebesgue point for $\theta$.

Thus, if we take into account (3.5) and (3.6), passing to the liminf as $\rho \rightarrow 0^{+}$in (3.4), we infer

$$
\begin{aligned}
f(x, A) & \leq \liminf _{\mathcal{R}_{x} \ni \rho \rightarrow 0^{+}} \frac{1}{\theta(x) \mathcal{H}^{k}\left(S \cap B_{\rho}(x)\right)} \int_{B} f\left(x, A+P_{\mu}(x) \nabla \varphi_{\rho}(y)\right) \mathrm{d} \mu^{x, \rho}(y) \\
& =\liminf _{\mathcal{R}_{x} \ni \rho \rightarrow 0^{+}} \frac{1}{\mu\left(B_{\rho}(x)\right)} \int_{B} f\left(x, A+P_{\mu}(x) \nabla \varphi_{\rho}(y)\right) \mathrm{d} \mu^{x, \rho}(y),
\end{aligned}
$$

as required.

\section{NECESSARY CONDITIONS FOR LOWER SEMICONTINUITY}

In this section, we study necessary conditions for the weak lower semicontinuity on $H_{\mu}^{1, p}\left(\mathbb{R}^{n}, \mathbb{R}^{m}\right)$ of the integral functional

$$
F(u):=\int_{\mathbb{R}^{n}} f\left(x, \nabla_{\mu} u\right) \mathrm{d} \mu, \quad u \in H_{\mu}^{1, p}\left(\mathbb{R}^{n}, \mathbb{R}^{m}\right),
$$

where the real function $f=f(x, z)$ is assumed to satisfy on $\mathbb{R}^{n} \times \mathbb{R}^{n m}$ the following conditions:

$f$ is $\mu$-measurable in $x$ and Lipschitz in $z$;

$f(x, z) \leq C\left(1+|z|^{p}\right)$ for some $C>0 ;$

$|f(x, z)-f(y, z)| \leq \eta(|y-x|)\left(1+|z|^{p}\right)$ for some function $\eta$ on $\mathbb{R}^{+}$continuous at 0.

We recall that we always make the assumption $p \in(1,+\infty)$. Moreover, we set for brevity $H_{\mu}^{1, p}:=H_{\mu}^{1, p}\left(\mathbb{R}^{n}, \mathbb{R}^{m}\right)$, and for any positive $\rho$ we let as above $\psi^{x, \rho}(y):=\rho \psi\left(\frac{y-x}{\rho}\right)$. 
Theorem 4.1. If the functional $F$ is weakly l.s.c. on $H_{\mu}^{1, p}$, then $f(x, \cdot)$ is $T_{\mu}(x)$-quasiconvex for $\mu$-a.e. $x$.

Proof. We proceed by contradiction, supposing that the $T_{\mu}(x)$-quasiconvexity is violated almost everywhere on a set $E$ of positive measure $\mu$. We can assume, without loss of generality, that every $x$ in $E$ is a Lebesgue point for the projector $P_{\mu}$, and that, for every $x \in E, \mu$ admits a nonzero measure $\nu_{x} \in \operatorname{Tan}(\mu, x)$ of product type; indeed each of these conditions is satisfied up to a $\mu$-negligible set.

Let $D$ be a dense subset of $\mathcal{L}\left(\mathbb{R}^{n}, \mathbb{R}^{m}\right)$. For $\mu$-a.e. $x \in E$, there exist $A_{x} \in D, \varphi_{x} \in \mathcal{D}\left(B, \mathbb{R}^{m}\right)$ and $k_{x} \in \mathbb{N}$ such that

$$
f\left(x, P_{\mu}(x) A_{x}\right) \geq \liminf _{\mathcal{R}_{x} \ni \rho \rightarrow 0^{+}} \frac{1}{\mu\left(B_{\rho}(x)\right)} \int_{B} f\left(x, P_{\mu}(x)\left[A_{x}+\nabla \varphi_{x}\right]\right) \mathrm{d} \mu^{x, \rho}+\frac{1}{k_{x}} .
$$

Indeed, if we could find a subset $E^{\prime}$ of $E$ with $\mu\left(E^{\prime}\right)>0$ where the opposite inequality holds for any $A \in D$, $k \in \mathbb{N}$ and $\varphi \in \mathcal{D}\left(B, \mathbb{R}^{m}\right)$, letting $k$ tend to $+\infty$, using the density of $D$ in $\mathcal{L}\left(\mathbb{R}^{n}, \mathbb{R}^{m}\right)$, and argueing as in the proof of Proposition 3.5, we would obtain that $f(x, \cdot)$ is $T_{\mu}(x)$-quasiconvex on $E^{\prime}$. Moreover, since the matrix $A_{x}$ and the integer $k_{x}$ in (4.5) vary in countable sets, possibly replacing $E$ by a smaller set we can suppose that they do not depend on $x$. We denote by $\left\{\rho_{i}\right\} \subset \mathcal{R}_{x}$ a sequence of radius tending to zero which achieves the liminf at the right hand side of (4.5), namely

$$
\liminf _{\mathcal{R}_{x} \ni \rho \rightarrow 0^{+}} \frac{1}{\mu\left(B_{\rho}(x)\right)} \int_{B} f\left(x, P_{\mu}(x)\left[A+\nabla \varphi_{x}\right]\right) \mathrm{d} \mu^{x, \rho}=\lim _{i \rightarrow+\infty} \frac{1}{\mu\left(B_{\rho_{i}}(x)\right)} \int_{B_{\rho_{i}}(x)} f\left(x, P_{\mu}(x)\left[A+\nabla \varphi_{x}^{x, \rho_{i}}\right]\right) \mathrm{d} \mu
$$

Since $f$ is Lipschitz in the second variable, and $x$ is a Lebesgue point for $P_{\mu}$, we have

$$
\begin{aligned}
& \int_{B_{\rho_{i}}(x)} f\left(x, P_{\mu}(x)\left[A+\nabla \varphi_{x}^{x, \rho_{i}}\right]\right) \mathrm{d} \mu-\int_{B_{\rho_{i}}(x)} f\left(x, P_{\mu}(y)\left[A+\nabla \varphi_{x}^{x, \rho_{i}}\right]\right) \mathrm{d} \mu \\
& \leq \operatorname{Lip}(f(x, \cdot)) \int_{B_{\rho_{i}}(x)}\left|\left[P_{\mu}(x)-P_{\mu}(y)\right]\left[A+\nabla \varphi_{x}^{x, \rho_{i}}\right]\right| \mathrm{d} \mu \\
& \leq \operatorname{Lip}(f(x, \cdot))\left(|A|+\left\|\nabla \varphi_{x}\right\|_{L_{\mu}^{\infty}(B)}\right) \int_{B_{\rho_{i}}(x)}\left|P_{\mu}(x)-P_{\mu}(y)\right| \mathrm{d} \mu=o\left(\mu\left(B_{\rho_{i}}(x)\right)\right)
\end{aligned}
$$

on the other hand, by (4.4) we obtain

$$
\begin{aligned}
& \int_{B_{\rho_{i}}(x)} f\left(x, P_{\mu}(y)\left[A+\nabla \varphi_{x}^{x, \rho_{i}}\right]\right) \mathrm{d} \mu-\int_{B_{\rho_{i}}(x)} f\left(y, P_{\mu}(y)\left[A+\nabla \varphi_{x}^{x, \rho_{i}}\right]\right) \mathrm{d} \mu \\
& \leq \int_{B_{\rho_{i}}(x)} \eta(|y-x|)\left(1+\left|A+\nabla \varphi_{x}^{x, \rho_{i}}\right|^{p}\right) \mathrm{d} \mu \\
& \leq \int_{B_{\rho_{i}}(x)} \eta(|y-x|)\left[1+2^{p-1}\left(|A|^{p}+\left\|\nabla \varphi_{x}\right\|_{L_{\mu}^{\infty}(B)}^{p}\right)\right] \mathrm{d} \mu=o\left(\mu\left(B_{\rho_{i}}(x)\right)\right)
\end{aligned}
$$

and, similarly,

$$
\begin{aligned}
& \mu\left(B_{\rho_{i}}(x)\right) f\left(x, P_{\mu}(x) A\right)-\int_{B_{\rho_{i}}(x)} f\left(y, P_{\mu}(y) A\right) \mathrm{d} \mu \\
& \leq \operatorname{Lip}(f(x, \cdot)) \int_{B_{\rho_{i}}(x)}\left|P_{\mu}(y)-P_{\mu}(x)\right||A| \mathrm{d} \mu+\int_{B_{\rho_{i}}(x)} \eta(|y-x|)\left(1+|A|^{p}\right) \mathrm{d} \mu=o\left(\mu\left(B_{\rho_{i}}(x)\right)\right) .
\end{aligned}
$$


Thus we infer from (4.5)

$$
\lim _{i \rightarrow+\infty} \frac{1}{\mu\left(B_{\rho_{i}}(x)\right)} \int_{B_{\rho_{i}}(x)} f\left(y, P_{\mu}(y) A\right) \mathrm{d} \mu \geq \lim _{i \rightarrow+\infty} \frac{1}{\mu\left(B_{\rho_{i}}(x)\right)} \int_{B_{\rho_{i}}(x)} f\left(y, P_{\mu}(y)\left[A+\nabla \varphi_{x}^{x, \rho_{i}}\right]\right) \mathrm{d} \mu+\frac{1}{k} .
$$

In particular we have, for $i$ sufficiently large,

$$
\int_{B_{\rho_{i}}(x)} f\left(y, P_{\mu}(y) A\right) \mathrm{d} \mu \geq \int_{B_{\rho_{i}}(x)} f\left(y, P_{\mu}(y)\left[A+\nabla \varphi_{x}^{x, \rho_{i}}\right]\right) \mathrm{d} \mu+\frac{\mu\left(B_{\rho_{i}}(x)\right)}{2 k} .
$$

Since $\partial B_{\rho_{i}}(x)$ is $\mu$-negligible, the same inequality holds as well if we replace the balls $B_{\rho_{i}}(x)$ by their closures $\bar{B}_{\rho_{i}}(x)$. Therefore, using the Vitali covering theorem, for every $n \in \mathbb{N}$ we can cover $E$ (up to a $\mu$-negligible set) by a countable family of disjoint closed balls $B_{j}:=\bar{B}_{\rho_{j}}\left(x_{j}\right)$, with $\rho_{j} \leq \frac{1}{n}$, such that (4.6) holds. We may assume in addition that

$$
\rho_{j} \leq \frac{1}{n\left\|\varphi_{x_{j}}\right\|_{L_{\mu}^{\infty}(B)}}, \quad \mu\left(B_{j}\right) \leq \frac{1}{j^{2}\left\|\nabla_{\mu} \varphi_{x_{j}}\right\|_{L_{\mu}^{\infty}(B)}^{p}}
$$

(the latter condition being satisfied for $\rho_{j}$ small enough, since the assumption $T_{\mu}(x) \neq\{0\} \mu$-a.e. implies that $\mu$ is atomless).

Consider then the sequence of functions

$$
u_{n}(y)= \begin{cases}A y+\varphi_{x_{j}}^{x_{j}, \rho_{j}}(y) & \text { if } y \in B_{j} \\ A y & \text { otherwise }\end{cases}
$$

and set $u(y)=A y$. By $(4.7), u_{n}$ converge weakly to $u$ in $H_{\mu}^{1, p}$, as

$$
\begin{aligned}
\left\|u_{n}-u\right\|_{L_{\mu}^{p}}^{p} & =\sum_{j}\left\|\varphi_{x_{j}}^{x_{j}, \rho_{j}}\right\|_{L_{\mu}^{p}\left(B_{j}\right)}^{p}=\sum_{j} \int_{B_{j}} \rho_{j}^{p}\left|\varphi_{x_{j}}\left(\frac{y-x_{j}}{\rho_{j}}\right)\right|^{p} \mathrm{~d} \mu \\
& \leq \sum_{j} \mu\left(B_{j}\right) \rho_{j}^{p}\left\|\varphi_{x_{j}}\right\|_{L_{\mu}^{\infty}(B)}^{p} \leq \frac{1}{n^{p}} \mu(E),
\end{aligned}
$$

and

$$
\begin{aligned}
\left\|\nabla_{\mu} u_{n}\right\|_{L_{\mu}^{p}}^{p} & =\sum_{j}\left\|\nabla_{\mu} \varphi_{x_{j}}^{x_{j}, \rho_{j}}\right\|_{L_{\mu}^{p}\left(B_{j}\right)}^{p}=\sum_{j} \int_{B_{j}}\left|\nabla_{\mu} \varphi_{x_{j}}\left[\frac{y-x_{j}}{\rho_{j}}\right]\right|^{p} \mathrm{~d} \mu \\
& \leq \sum_{j} \mu\left(B_{j}\right)\left\|\nabla_{\mu} \varphi_{x_{j}}\right\|_{L_{\mu}^{\infty}(B)}^{p} \leq \sum_{j} \frac{1}{j^{2}} .
\end{aligned}
$$

Now, summing over the index $j$ the inequalities (4.6) on $B_{j}$, we get

$$
F(u) \geq F\left(u_{n}\right)+\frac{\mu(E)}{2 k}>F\left(u_{n}\right)
$$

in particular, passing to the liminf as $n \rightarrow+\infty$, this contradicts the lower semicontinuity of $F$.

In order to prove that the $\mu$-quasiconvexity by blow-up of $f$ is also necessary for lower semicontinuity, we are led to require a Poincaré-type inequality for $\mu$. Similarly as in [19] (Sect. 2), we say that $\mu$ enjoys the $p$-Poincaré 
inequality if there exist some constants $M>0$ and $\sigma \geq 1$ such that, for $\mu$-a.e. point $x$, it holds

$$
\int_{B_{\rho}(x)}|\varphi-\bar{\varphi}|^{p} \mathrm{~d} \mu \leq M \rho^{p} \int_{B_{\sigma \rho}(x)}\left|\nabla_{\mu} \varphi\right|^{p} \mathrm{~d} \mu \quad \forall \rho>0, \forall \varphi \in H_{\mu}^{1, p}
$$

where $\bar{\varphi}:=f_{B_{\rho}(x)} \varphi \mathrm{d} \mu$.

Theorem 4.2. Let $\mu$ satisfy the $p$-Poincaré inequality. If the functional $F$ is weakly l.s.c. on $H_{\mu}^{1, p}$, then $f(x, \cdot)$ is $\mu$-quasiconvex by blow-up for $\mu$-a.e. $x$.

Proof. We argue by contradiction, assuming that the $\mu$-quasiconvexity by blow-up of $f$ is violated on a subset $E \subset \mathbb{R}^{n}$ of positive measure $\mu$. Similarly as in the proof of Theorem 4.1, we find that there exist $A \in \mathcal{L}\left(\mathbb{R}^{n}, \mathbb{R}^{m}\right)$ and $k \in \mathbb{N}$ such that, for $\mu$-a.e. $x \in E$, the inequality

$$
f\left(x, P_{\mu}(x) A\right) \geq \liminf _{\mathcal{R}_{x} \ni \rho \rightarrow 0^{+}} \frac{1}{\mu\left(B_{\rho}(x)\right)} \int_{B} f\left(x, P_{\mu}(x)\left[A+\nabla \varphi_{x, \rho}\right]\right) \mathrm{d} \mu^{x, \rho}+\frac{1}{k}
$$

holds for some admissible family $\left\{\varphi_{x, \rho}\right\} \subset \mathcal{F}_{x}$. We stress that now such family a priori do depends on $\rho$, differently from what happened in (4.5). Possibly replacing $E$ with a smaller set, we may assume that (4.8) holds at every $x \in E$, and we may also find an integer $h$ such that

$$
\sup _{\rho \in \mathcal{R}_{x}} \frac{1}{\mu\left(B_{\rho}(x)\right)} \int_{B}\left|\nabla \varphi_{x, \rho}\right|^{p} \mathrm{~d} \mu^{x, \rho}<h \quad \forall x \in E .
$$

Then, following the same line of proof of Theorem 4.1, we obtain that, for a suitable sequence of radius $\left\{\rho_{i}\right\} \subset \mathcal{R}_{x}$, definitively in $i$ it holds

$$
\int_{\bar{B}_{\rho_{i}}(x)} f\left(y, P_{\mu}(y) A\right) \mathrm{d} \mu \geq \int_{\bar{B}_{\rho_{i}}(x)} f\left(y, P_{\mu}(y)\left[A+\nabla \varphi_{x, \rho_{i}}^{x, \rho_{i}}\right]\right) \mathrm{d} \mu+\frac{\mu\left(\bar{B}_{\rho_{i}}(x)\right)}{2 k}
$$

(here the subscript ${ }_{x, \rho_{i}}$ and the superscript ${ }^{x, \rho_{i}}$ denote respectively the dependence of $\varphi$ on the point $x$ and on the radius $\rho_{i}$, and the blow-up at $x$ of factor $\rho_{i}$, namely $\left.\varphi_{x, \rho_{i}}^{x, \rho_{i}}(y)=\rho_{i} \varphi_{x, \rho_{i}}\left(\frac{y-x}{\rho_{i}}\right)\right)$.

For every $n \in \mathbb{N}$, consider a countable covering of $\mu$-almost all $E$ made of disjoint closed balls $B_{j}:=\bar{B}_{\rho_{j}}\left(x_{j}\right)$ of radius $\rho_{j}<\frac{1}{n}$ such that (4.10) holds. Let us consider the sequence of functions

$$
u_{n}(y)= \begin{cases}A y+\varphi_{x_{j}}^{x_{j}, \rho_{j}}(y)-\bar{\varphi}_{j} & \text { if } y \in B_{j} \\ A y & \text { otherwise }\end{cases}
$$

where $\bar{\varphi}_{j}:=f_{B_{j}} \varphi_{x_{j}, \rho_{j}}^{x_{j}, \rho_{j}}(y) \mathrm{d} \mu(y)$.

We claim that $\left\{u_{n}\right\}$ converges weakly to $u(y)=A y$ in $H_{\mu}^{1, p}$. Indeed, by (4.9), we have

$$
\begin{aligned}
\left\|\nabla_{\mu} u_{n}\right\|_{L_{\mu}^{p}}^{p} & =\sum_{j}\left\|\nabla_{\mu} \varphi_{x_{j}, \rho_{j}}^{x_{j}, \rho_{j}}\right\|_{L_{\mu}^{p}\left(B_{j}\right)}^{p}=\sum_{j} \int_{B_{j}}\left|\nabla_{\mu} \varphi_{x_{j}, \rho_{j}}\left[\frac{y-x_{j}}{\rho_{j}}\right]\right|^{p} \mathrm{~d} \mu \\
& =\sum_{j} \int_{B}\left|\nabla_{\mu} \varphi_{x_{j}, \rho_{j}}\right|^{p} \mathrm{~d} \mu^{x_{j}, \rho_{j}} \leq h \mu(E) .
\end{aligned}
$$


Moreover, via the $p$-Poincaré inequality for $\mu$, we have

$$
\begin{aligned}
\left\|u_{n}-u\right\|_{L_{\mu}^{p}}^{p} & =\sum_{j}\left\|\varphi_{x_{j}, \rho_{j}}^{x_{j}, \rho_{j}}-\bar{\varphi}_{j}\right\|_{L_{\mu}^{p}\left(B_{j}\right)}^{p} \leq M \sum_{j} \rho_{j}^{p}\left\|\nabla_{\mu} \varphi_{x_{j}, \rho_{j}}^{x_{j}, \rho_{j}}\right\|_{L_{\mu}^{p}\left(\sigma B_{j}\right)}^{p} \\
& =M \sum_{j} \rho_{j}^{p} \int_{\sigma B_{j}}\left|\nabla_{\mu} \varphi_{x_{j}, \rho_{j}}\left[\frac{y-x_{j}}{\rho_{j}}\right]\right|^{p} \mathrm{~d} \mu=M \sum_{j} \rho_{j}^{p} \int_{\sigma B}\left|\nabla_{\mu} \varphi_{x_{j}, \rho_{j}}\right|^{p} \mathrm{~d} \mu^{x_{j}, \rho_{j}} \\
& =M \sum_{j} \rho_{j}^{p} \int_{B}\left|\nabla_{\mu} \varphi_{x_{j}, \rho_{j}}\right|^{p} \mathrm{~d} \mu^{x_{j}, \rho_{j}} \leq \frac{M h \mu(E)}{n^{p}} .
\end{aligned}
$$

Therefore, summing over the index $j$ the inequalities (4.10) on $B_{j}$, leads to contradict the lower semicontinuity of $F$.

Corollary 4.3. Let $\mu$ satisfy the p-Poincaré inequality. If $f(x, \cdot)$ is convex for $\mu$-a.e. $x$, then it is $\mu$-quasiconvex by blow-up for $\mu$-a.e. $x$. In particular, in the scalar case $m=1$, the $\mu$-quasiconvexity by blow-up of $f(x, \cdot) \mu$ a.e. is equivalent to its $T_{\mu}$-quasiconvexity $\mu$-a.e.

Proof. If $f(x, \cdot)$ is convex for $\mu$-a.e. $x$, also the function $\tilde{f}(x, z):=f\left(x, P_{\mu}(x) z\right)$ is convex in the variable $z$ for $\mu$-a.e. $x$. Applying the relaxation result proved in [9] (Th. 3.1), since $\inf \left\{\tilde{f}(x, z+\underset{\tilde{F}}{\tilde{f}}): \operatorname{ker} \xi \supseteq T_{\mu}(x)\right\}=\tilde{f}(x, z)$, we obtain the lower semicontinuity of the functional $\widetilde{F}(u)=\int \tilde{f}\left(x, \nabla_{\mu} u\right) \mathrm{d} \mu$. As $\widetilde{F} \equiv F$, by Theorem $4.2 f(x, \cdot)$ turns out to be $\mu$-quasiconvex by blow-up for $\mu$-a.e. $x$. The second part of the statement follows straightforward, taking into account Remark 3.2.

\section{SUFFiCIENT CONDITIONS FOR LOWER SEMICONTINUITY}

We are going to investigate sufficiency conditions for the lower semicontinuity of the functional $F$ defined by (4.1). We still assume that the integrand $f$ suits assumptions (4.2-4.4); in addition, we suppose from now on that it satisfies a growth condition from below of the kind

$$
f(x, z) \geq-c\left(1+|z|^{q}\right)
$$

for some positive constant $c$, and some exponent $q \in[1, p)$.

We say that $\mu$ enjoys the doubling property if there exists a positive constant $D$ such that, for $\mu$-a.e. $x$, it holds

$$
\mu\left(B_{2 \rho}(x)\right) \leq D \mu\left(B_{\rho}(x)\right) \quad \forall \rho>0
$$

The main results of this section are the following:

Theorem 5.1. Let $\mu$ satisfy the $p$-Poincaré inequality and the doubling condition. If $f(x, \cdot)$ is $\mu$-quasiconvex by blow-up for $\mu$-a.e. $x$, then the functional $F$ is weakly l.s.c. on $H_{\mu}^{1, p}$.

Theorem 5.2. Let $\mu$ be the weight measure of a $k$-rectifiable varifold with bounded first variation, and assume that $\mu$ satisfies the p-Poincaré inequality. If $f(x, \cdot)$ is $T_{\mu}(x)$-quasiconvex at $\mu . a . e x$, then the functional $F$ is weakly l.s.c. on $H_{\mu}^{1, p}$.

We observe that Theorem 5.2 is an immediate consequence of Theorem 5.1 and Proposition 3.6 (indeed, the doubling property is enjoyed by any measure $\mu$ as in the assumptions of Th. 5.2). Before proving Theorem 5.1, we state two preliminary lemmas. 
Lemma 5.3. Let $\left\{u_{h}\right\}$ be a bounded sequence in $H_{\mu}^{1, p}$. For any ball $B$ centered at a point $x$ where both (4.8) and (5.2) hold, we can extract a subsequence which converges strongly in $L_{\mu}^{p}(B)$.

Proof. It is an immediate consequence of Theorem 8.3 in [19] (cf. also [20]).

Lemma 5.4. Let $u \in H_{\mu}^{1, p}$, let $x$ be a Lebesgue point for $\nabla_{\mu} u$ and $P_{\mu}$ where both (4.8) and (5.2) hold, and let $\left\{\rho_{i}\right\}$ be a sequence of radius tending to zero. Setting

$$
w_{i}(y):=\frac{u\left(x+\rho_{i} y\right)-\nabla_{\mu} u(x) \rho_{i} y-a_{i}}{\rho_{i}}, \quad a_{i}:=f_{B_{\rho_{i}}(x)}\left[u(y)-\nabla_{\mu} u(x)(y-x)\right] \mathrm{d} \mu(y),
$$

we have

$$
\int_{B}\left|w_{i}\right|^{p} \mathrm{~d} \mu^{x, \rho_{i}}=o\left(\mu\left(B_{\rho_{i}}(x)\right)\right)
$$

Proof. Using in the order (4.8), that $x$ is a Lebesgue point for $\nabla_{\mu} u$ and $P_{\mu}$, and (5.2), we infer

$$
\begin{aligned}
& \lim _{i} \frac{1}{\mu\left(B_{\rho_{i}}(x)\right)} \int_{B}\left|w_{i}\right|^{p} \mathrm{~d} \mu^{x, \rho_{i}} \\
& \lim _{i} \frac{1}{\mu\left(B_{\rho_{i}}(x)\right)} \int_{B_{\rho_{i}}(x)} \frac{\left|u(y)-\nabla_{\mu} u(x)(y-x)-a_{i}\right|^{p}}{\rho_{i}^{p}} \mathrm{~d} \mu(y) \\
\leq & \lim _{i} \frac{M}{\mu\left(B_{\rho_{i}}(x)\right)} \int_{B_{\sigma \rho_{i}}(x)}\left|\nabla_{\mu} u(y)-P_{\mu}(y) \nabla_{\mu} u(x)\right|^{p} \mathrm{~d} \mu(y) \\
\leq & \lim _{i} \frac{M 2^{p-1}}{\mu\left(B_{\rho_{i}}(x)\right)} \int_{B_{\sigma \rho_{i}}(x)}\left|\nabla_{\mu} u(y)-\nabla_{\mu} u(x)\right|^{p}+\left|\left(P_{\mu}(y)-P_{\mu}(x)\right) \nabla_{\mu} u(x)\right|^{p} \mathrm{~d} \mu(y) \\
\leq & M 2^{p-1} \sup _{i} \frac{\mu\left(B_{\sigma \rho_{i}}(x)\right)}{\mu\left(B_{\rho_{i}}(x)\right)} \lim _{i} \frac{1}{\mu\left(B_{\sigma \rho_{i}(x)}\right)} \int_{B_{\sigma \rho_{i}}(x)}\left|\nabla_{\mu} u(y)-\nabla_{\mu} u(x)\right|^{p}+
\end{aligned}
$$

We are now in a position to give the proof of Theorem 5.1. For convenience, it will be divided into several steps.

Proof of Theorem 5.1 .

Step 1: reduction to nonnegative $f$.

We claim that, due to the growth condition (5.1), it is not restrictive to assume that $f$ is nonnegative. Indeed, suppose the statement true when $f$ is nonnegative (hence when $\inf f>-\infty$ ), and let $u_{h} \rightarrow u$ in $H_{\mu}^{1, p}$. Letting, for any $k \in \mathbf{Z}^{-}, F_{k}$ be the functional defined in (4.1) where $f$ is replaced by $f_{k}:=\max \{f, k\}$, we have:

$$
F(u) \leq F_{k}(u) \leq \liminf _{h \rightarrow+\infty} F_{k}\left(u_{h}\right) .
$$

Passing to the limit as $k \rightarrow-\infty$, we get the required lower semicontinuity inequality for $F$ provided $F_{k}\left(u_{h}\right)$ converge to $F\left(u_{h}\right)$ as $k \rightarrow-\infty$ uniformly in $h$. Actually, setting $A_{h, k}:=\left\{x: f\left(x, \nabla_{\mu} u_{h}(x)\right) \leq k\right\}$ it holds:

$$
\begin{aligned}
\left|F_{k}\left(u_{h}\right)-F\left(u_{h}\right)\right| & \leq \int\left|f_{k}-f\right|\left(x, \nabla_{\mu} u_{h}\right) \mathrm{d} \mu=\int_{A_{h, k}} k-f\left(x, \nabla_{\mu} u_{h}\right) \mathrm{d} \mu \leq c \int_{A_{h, k}}\left(1+\left|\nabla_{\mu} u_{h}\right|^{q}\right) \mathrm{d} \mu \\
& \leq c \mu\left(A_{h, k}\right)+c\left(\int\left|\nabla_{\mu} u_{h}\right|^{p} \mathrm{~d} \mu\right)^{\frac{q}{p}} \mu\left(A_{h, k}\right)^{\frac{p-q}{p}} .
\end{aligned}
$$


Since $\left\{u_{h}\right\}$ is bounded in $H_{\mu}^{1, p}$, it is enough to check that $\lim _{k \rightarrow+\infty} \mu\left(A_{h, k}\right)=0$ uniformly in $h$. We have indeed

$$
\begin{aligned}
\mu\left(A_{h, k}\right) & \leq \frac{1}{k} \int_{A_{h, k}} f\left(x, \nabla_{\mu} u_{h}\right) \mathrm{d} \mu \leq-\frac{c}{k} \int_{A_{h, k}}\left(1+\left|\nabla_{\mu} u_{h}\right|^{q}\right) \mathrm{d} \mu \\
& \leq-\frac{c}{k}\left[\mu\left(A_{h, k}\right)+\left(\int\left|\nabla_{\mu} u_{h}\right|^{p} \mathrm{~d} \mu\right)^{\frac{q}{p}} \mu\left(A_{h, k}\right)^{\frac{p-q}{p}}\right] \leq-\frac{c}{k}\left[\mu\left(A_{h, k}\right)+\Lambda \mu\left(A_{h, k}\right)^{\frac{p-q}{p}}\right]
\end{aligned}
$$

(here and in the following, $\Lambda$ denotes a generic positive constant). Since we can assume that $\mu\left(A_{h, k}\right)>0$, we can divide by $\mu\left(A_{h, k}\right)$ and we obtain

$$
1 \leq-\frac{c}{k}\left[1+\Lambda \mu\left(A_{h, k}\right)^{-\frac{q}{p}}\right]
$$

therefore

$$
\mu\left(A_{h, k}\right) \leq\left[-\frac{c \Lambda}{k+c}\right]^{\frac{p}{q}}
$$

which entails $\lim _{k \rightarrow-\infty} \mu\left(A_{h, k}\right)=0$ uniformly in $h$.

Step 2: reduction to smooth sequences.

We claim that it is enough to prove the inequality $F(u) \leq \liminf _{h} F\left(u_{h}\right)$ when the functions $\left\{u_{h}\right\}$ (weakly converging to $u$ in $\left.H_{\mu}^{1, p}\right)$ belong to $\mathcal{D}\left(\mathbb{R}^{n}, \mathbb{R}^{m}\right)$, and satisfy the boundedness estimate $\sup _{h} \int\left|\nabla u_{h}\right|^{p} \mathrm{~d} \mu<+\infty$. Indeed, let us show that, for any sequence $\left\{u_{h}\right\} \subset H_{\mu}^{1, p}$ weakly converging to $u$, with liminf $F\left(u_{h}\right)<+\infty$, we can find another sequence $\left\{\tilde{u}_{h}\right\} \subset \mathcal{D}\left(\mathbb{R}^{n}, \mathbb{R}^{m}\right)$, still weakly converging to $u$, such that $\liminf _{h} F\left(u_{h}\right)=\liminf F\left(\tilde{u}_{h}\right)$ and $\sup _{h} \int\left|\nabla \tilde{u}_{h}\right|^{p} \mathrm{~d} \mu<+\infty$. For any fixed $h$, let $\left\{u_{h, k}\right\}_{k} \subset \mathcal{D}\left(\mathbb{R}^{n}, \mathbb{R}^{m}\right)$ be a sequence converging (strongly) to $u_{h}$ in $H_{\mu}^{1, p}$ and such that $\lim _{k} \int\left|\nabla u_{h, k}\right|^{p} \mathrm{~d} \mu=\int\left|\nabla_{\mu} u_{h}\right|^{p} \mathrm{~d} \mu$; such a sequence exists by (2.2). Then $\left\{F\left(u_{h, k}\right)\right\}$ converges to $F\left(u_{h}\right)$ as $k \rightarrow+\infty$ (to see that, it is enough to notice that the functional $u \mapsto C \int\left(1+\left|\nabla_{\mu} u\right|^{p}\right) \mathrm{d} \mu$ is strongly continuous on $H_{\mu}^{1, p}$ and to apply Fatou's lemma separately to the sequences $\int f\left(x, \nabla_{\mu} u_{h, k}\right) \mathrm{d} \mu$ and $\left.\int\left[C\left(1+\left|\nabla_{\mu} u_{h, k}\right|^{p}\right)-f\left(x, \nabla_{\mu} u_{h, k}\right)\right] \mathrm{d} \mu\right)$. So, for any $h$, we can find an index $k(h)$ such that

$$
\left\|u_{h, k(h)}-u_{h}\right\|_{1, p, \mu} \leq \frac{1}{h}, \quad\left|F\left(u_{h, k(h)}\right)-F\left(u_{h}\right)\right| \leq \frac{1}{h}, \quad \int\left|\nabla u_{h, k(h)}\right|^{p} \mathrm{~d} \mu-\int\left|\nabla_{\mu} u_{h}\right|^{p} \mathrm{~d} \mu \leq \frac{1}{h} .
$$

Then our claim holds taking $\tilde{u}_{h}=u_{h, k(h)}$.

Summing up, we may assume that $f \geq 0$, and that $\left\{u_{h}\right\} \subset \mathcal{D}\left(\mathbb{R}^{n}, \mathbb{R}^{m}\right)$ converge weakly to $u$ in $H_{\mu}^{1, p}$, with $\liminf _{h} F\left(u_{h}\right)=\lim _{h} F\left(u_{h}\right)<+\infty$, and $\sup _{h} \int\left|\nabla u_{h}\right|^{p} \mathrm{~d} \mu<+\infty$.

\section{Step 3: localization.}

Up to subsequences, the measures $\lambda_{h}:=f\left(y, \nabla_{\mu} u_{h}(y)\right) \mathrm{d} \mu(y)$ converge weakly to some positive measure $\lambda$. By the Radon-Nikodym theorem, we can decompose $\lambda$ as $\lambda=\lambda_{a}+\lambda_{s}$, with $\lambda_{a}$ and $\lambda_{s}$ respectively absolutely continuous and singular with respect to $\mu$. Then the required inequality $F(u) \leq \lim _{h} F\left(u_{h}\right)$ will follow from the lower semicontinuity of the total variation with respect to the weak (star) convergence of measures, if we prove that $\lambda_{a} \geq f\left(y, \nabla_{\mu} u(y)\right) \mathrm{d} \mu(y)$. The Besicovitch derivation theorem ensures that such inequality is satisfied 
provided

$$
\lim _{\rho \rightarrow 0} \frac{\lambda\left(B_{\rho}(x)\right)}{\int_{B_{\rho}(x)} f\left(y, \nabla_{\mu} u(y)\right) \mathrm{d} \mu(y)} \geq 1 \quad \text { for } \mu \text {-a.e. } x
$$

(the limit at the left hand side existing finite for $\mu$-a.e. $x$ [15], 1.6).

Thus, the remaining of the proof will be devoted to show the inequality (5.4). It is not restrictive to do that at a given point $x$ where some properties which are satisfied $\mu$-a.e. hold. More precisely, we fix a point $x$ such that

$-T_{\mu}(x) \neq\{0\}$

- (4.8) and (5.2) hold at $x$;

- $f(x, \cdot)$ is $\mu$-quasiconvex by blow-up;

- the limit at the left hand side of (5.4) exists and it is finite;

$-x$ is a Lebesgue point for both $P_{\mu}$ and $\nabla_{\mu} u$;

- letting $\sigma:=\left|\nabla_{\mu} u(y)\right|^{p} \mathrm{~d} \mu(y)$, then $D_{\mu} \sigma(x):=\lim _{\rho \rightarrow 0} \frac{\sigma\left(B_{\rho}(x)\right)}{\mu\left(B_{\rho}(x)\right)}$ exists and it is finite;

- if $\tau$ is (possibly passing to a subsequence) the weak limit of the measures $\tau_{h}:=\left|\nabla u_{h}(y)\right|^{p} \mathrm{~d} \mu(y)$, then $D_{\mu} \tau:=\lim _{\rho \rightarrow 0} \frac{\tau\left(B_{\rho}(x)\right)}{\mu\left(B_{\rho}(x)\right)}$ exists and it is finite.

\section{Step 4: blow-up.}

For $x$ chosen as above, we can now select a sequence of radius $\left\{\rho_{i}\right\} \subset \mathcal{R}_{x}$ tending to zero such that the blownup measures $\frac{\mu_{x, \rho_{i}}}{\mu\left(B_{\rho_{i}}(x)\right)}$ converge weakly to some (nonzero) element $\nu \in \operatorname{Tan}(\mu, x)$. We then choose $R \in(0,1)$ such that $\nu\left(\partial B_{R}(x)\right)=0$, and, setting $t_{i}:=R \rho_{i}$,

$$
\lambda\left(\partial B_{t_{i}}(x)\right)=\mu\left(\partial B_{t_{i}}(x)\right)=\tau\left(\partial B_{t_{i}}(x)\right)=0 \quad \forall i .
$$

We are done if we show that

$$
\lim _{i} \frac{\lambda\left(B_{t_{i}}(x)\right)}{\int_{B_{t_{i}}(x)} f\left(y, \nabla_{\mu} u(y)\right) \mathrm{d} \mu(y)} \geq 1
$$

Since $\lambda\left(\partial B_{t_{i}}(x)\right)=\mu\left(\partial B_{t_{i}}(x)\right)=0$, by the weak convergence of $\lambda_{h}$ to $\lambda$ it holds

$$
\lambda\left(B_{t_{i}}(x)\right)=\lambda\left(\bar{B}_{t_{i}}(x)\right) \geq \underset{h}{\limsup } \lambda_{h}\left(\bar{B}_{t_{i}}(x)\right)=\underset{h}{\limsup } \lambda_{h}\left(B_{t_{i}}(x)\right) .
$$

Then, to get (5.5), it is sufficient to prove that

$$
\underset{i}{\limsup } \underset{h}{\limsup } \frac{\int_{B_{t_{i}}(x)} f\left(y, \nabla_{\mu} u_{h}(y)\right) \mathrm{d} \mu(y)}{\int_{B_{t_{i}}(x)} f\left(y, \nabla_{\mu} u(y)\right) \mathrm{d} \mu(y)} \geq 1 .
$$

We observe that the latter two conditions imposed on $x$ in Step 3 imply respectively the existence of some positive constant $\Lambda$ such that, for $i$ large enough and $h \geq \bar{h}(i)$,

$$
\int_{B_{t_{i}}(x)}\left|\nabla_{\mu} u\right|^{p} \mathrm{~d} \mu \leq \Lambda \mu\left(B_{t_{i}}(x)\right), \quad \int_{B_{t_{i}}(x)}\left|\nabla u_{h}\right|^{p} \mathrm{~d} \mu \leq \Lambda \mu\left(B_{t_{i}}(x)\right) .
$$


Indeed, the former inequality is immediate. For the latter, it holds if $\bar{h}(i)$ is chosen in order that, for some $\omega_{i}=o\left(\mu\left(B_{t_{i}}(x)\right)\right)$,

$$
\tau_{h}\left(B_{t_{i}}(x)\right) \leq \tau\left(B_{t_{i}}(x)\right)+\omega_{i} \quad \forall h \geq \bar{h}(i)
$$

such choice of $\bar{h}(i)$ is made possible by the assumption that $\partial B_{t_{i}}(x)$ are $\tau_{h}$ and $\tau$-negligible, which gives $\tau\left(B_{t_{i}}(x)\right) \geq \limsup \tau_{h}\left(B_{t_{i}}(x)\right)$.

Possibly taking $\bar{h}(i)$ larger, by Lemma 5.3, we may also assume that, letting $\omega_{i}$ as above and $a_{i}$ as in (5.3), for $h \geq \bar{h}(i)$ it holds

$$
\int_{B_{\rho_{i}}(x)} \frac{\left|u_{h}(y)-a_{i}-\nabla_{\mu} u(x)(y-x)\right|^{p}}{\rho_{i}^{p}} \mathrm{~d} \mu(y) \leq \omega_{i}+\int_{B_{\rho_{i}}(x)} \frac{\left|u(y)-a_{i}-\nabla_{\mu} u(x)(y-x)\right|^{p}}{\rho_{i}^{p}} \mathrm{~d} \mu(y) .
$$

Setting for brevity $u_{i}:=u_{\bar{h}(i)}$, we shall have (5.6) if we prove that

$$
\limsup _{i} \frac{\int_{B_{t_{i}}(x)} f\left(y, \nabla_{\mu} u_{i}(y)\right) \mathrm{d} \mu(y)}{\int_{B_{t_{i}}(x)} f\left(y, \nabla_{\mu} u(y)\right) \mathrm{d} \mu(y)} \geq 1
$$

Step 5: freezing the variable in $f(\cdot, z)$ and $P_{\mu}(\cdot)$.

Using the Lipschitz assumption on $f$, that $x$ is a Lebesgue point $\nabla_{\mu} u,(4.4)$, and (5.7), we get

$$
\begin{aligned}
& \int_{B_{t_{i}}(x)}\left[f\left(y, \nabla_{\mu} u(y)\right)-f\left(x, \nabla_{\mu} u(x)\right)\right] \mathrm{d} \mu(y) \\
& \leq \operatorname{Lip}(f(x, \cdot)) \int_{B_{t_{i}}(x)}\left|\nabla_{\mu} u(y)-\nabla_{\mu} u(x)\right| \mathrm{d} \mu(y) \\
& +\int_{B_{t_{i}}(x)} \eta(|y-x|)\left(1+\left|\nabla_{\mu} u\right|^{p}\right) \mathrm{d} \mu(y)=o\left(\mu\left(B_{t_{i}}(x)\right)\right)
\end{aligned}
$$

similarly, by (4.4) and (5.7),

$$
\begin{aligned}
& \int_{B_{t_{i}}(x)} f\left(y, \nabla_{\mu} u_{i}(y)\right) \mathrm{d} \mu(y)-\int_{B_{t_{i}}(x)} f\left(x, \nabla_{\mu} u_{i}(y)\right) \mathrm{d} \mu(y) \\
\leq & \int_{B_{t_{i}}(x)} \eta(|y-x|)\left(1+\left|\nabla_{\mu} u_{i}(y)\right|^{p}\right) \mathrm{d} \mu(y)=o\left(\mu\left(B_{t_{i}}(x)\right)\right) ;
\end{aligned}
$$

finally, using the Lipschitz assumption on $f$, that $x$ is a Lebesgue point for $P_{\mu}$ and again (5.7),

$$
\begin{aligned}
& \int_{B_{t_{i}}(x)} f\left(x, \nabla_{\mu} u_{i}(y)\right) \mathrm{d} \mu(y)-\int_{B_{t_{i}}(x)} f\left(x, P_{\mu}(x) \nabla u_{i}(y)\right) \mathrm{d} \mu(y) \\
& \leq \operatorname{Lip}(f(x, \cdot))\left[\int_{B_{t_{i}}(x)}\left|P_{\mu}(y)-P_{\mu}(x)\right|^{p^{\prime}} \mathrm{d} \mu(y)\right]^{\frac{1}{p^{\prime}}}\left[\int_{B_{t_{i}}(x)}\left|\nabla u_{i}(y)\right|^{p} \mathrm{~d} \mu(y)\right]^{\frac{1}{p}} \\
& =\operatorname{Lip}(f(x, \cdot)) o\left(\mu\left(B_{t_{i}}(x)\right)\right)^{\frac{1}{p^{\prime}}}\left[\int_{B_{t_{i}}(x)}\left|\nabla u_{i}(y)\right|^{p} \mathrm{~d} \mu(y)\right]^{\frac{1}{p}}=o\left(\mu\left(B_{t_{i}}(x)\right) .\right.
\end{aligned}
$$


Then (5.9) can be rewritten as

$$
\underset{i}{\limsup } \frac{1}{\mu\left(B_{t_{i}}(x)\right)} \int_{B_{t_{i}}(x)} f\left(x, P_{\mu}(x) \nabla u_{i}(y)\right) \mathrm{d} \mu(y) \geq f\left(x, \nabla_{\mu} u(x)\right),
$$

or equivalently

$$
\limsup _{i} \frac{1}{\mu\left(B_{t_{i}}(x)\right)} \int_{B} f\left(x, A+P_{\mu}(x) \nabla v_{i}\right) \mathrm{d} \mu^{x, t_{i}} \geq f(x, A)
$$

where

$$
v_{i}(y):=\frac{u_{i}\left(x+t_{i} y\right)-a_{i}-A t_{i} y}{t_{i}}, \quad A:=\nabla_{\mu} u(x) .
$$

Step 6: setting an affine datum on the boundary.

For any integer $K$, and for any $k=1, \ldots, K$, let $B_{k}$ be the ball, centered at 0 , of radius $r_{k}:=r+\frac{k}{K}(R-r)$, $r \in(0, R)$. Let $\varphi_{k}$ be a smooth function with values into [0,1], holding 1 on $B_{k-1}, 0$ outside $B_{k}$, and satisfying $\left|\nabla \varphi_{k}\right| \leq \Lambda K$ for some positive constant $\Lambda$.

For each $k=1, \ldots, K$, the family $\left\{v_{i}^{R} \varphi_{k}\right\}_{\rho_{i}} \subset \mathcal{D}\left(B, \mathbb{R}^{m}\right)$ is admissible by blow-up at $x$, where

$$
v_{i}^{R}(y):=R v_{i}\left(\frac{y}{R}\right)=\frac{u_{i}\left(x+\rho_{i} y\right)-a_{i}-A \rho_{i} y}{\rho_{i}} .
$$

Indeed, using (5.8) and Lemma 5.4, we obtain

$$
\begin{aligned}
& \left(\int_{B}\left|\nabla\left(v_{i}^{R} \varphi_{k}\right)\right|^{p} \mathrm{~d} \mu^{x, \rho_{i}}\right)^{\frac{1}{p}} \leq\left(\int_{B} \Lambda^{p} K^{p}\left|v_{i}^{R}\right|^{p} \mathrm{~d} \mu^{x, \rho_{i}}\right)^{\frac{1}{p}}+\left(\int_{B}\left|\nabla v_{i}^{R}\right|^{p} \mathrm{~d} \mu^{x, \rho_{i}}\right)^{\frac{1}{p}} \\
= & \Lambda K\left(\int_{B_{\rho_{i}}(x)} \frac{\left|u_{i}(y)-a_{i}-A(y-x)\right|^{p}}{\rho_{i}^{p}} \mathrm{~d} \mu(y)\right)^{\frac{1}{p}}+\left(\int_{B_{\rho_{i}}(x)}\left|\nabla u_{i}(y)-A\right|^{p} \mathrm{~d} \mu(y)\right)^{\frac{1}{p}} \\
\leq & \Lambda K o\left(\mu\left(B_{\rho_{i}}(x)\right)^{\frac{1}{p}}\right)+\left(\Lambda^{\frac{1}{p}}+|A|\right) \mu\left(B_{\rho_{i}}(x)\right)^{\frac{1}{p}} .
\end{aligned}
$$

Step 7: conclusion via $\mu$-quasiconvexity by blow-up.

Since $f(x, \cdot)$ is $\mu$-quasiconvex by blow-up, setting $v_{i, k}^{R}:=v_{i}^{R} \varphi_{k}$, we have

$$
\underset{i}{\limsup } \frac{1}{\mu\left(B_{\rho_{i}}(x)\right)} \int_{B} f\left(x, A+P_{\mu}(x) \nabla v_{i, k}^{R}\right) \mathrm{d} \mu^{x, \rho_{i}} \geq f(x, A) .
$$

So, for each $k$ and each $\alpha>1$ we can find a subsequence of radius $\rho_{i}$ (not relabeled) such that

$$
\begin{aligned}
f(x, A) \mu\left(B_{\rho_{i}}(x)\right) \leq & \int_{B} \alpha f\left(x, A+P_{\mu}(x) \nabla v_{i, k}^{R}\right) \mathrm{d} \mu^{x, \rho_{i}} \\
= & \int_{B \backslash B_{k}} \alpha f(x, A) \mathrm{d} \mu^{x, \rho_{i}}+\int_{B_{k} \backslash B_{k-1}} \alpha f\left(x, A+P_{\mu}(x) \nabla v_{i, k}^{R}\right) \mathrm{d} \mu^{x, \rho_{i}} \\
& +\int_{B_{k-1}} \alpha f\left(x, A+P_{\mu}(x) \nabla v_{i}^{R}\right) \mathrm{d} \mu^{x, \rho_{i}} .
\end{aligned}
$$


Recalling that $f$ is nonnegative and satisfies (4.3) we infer

$$
\begin{aligned}
& (1-\alpha) f(x, A) \mu\left(B_{\rho_{i}}(x)\right)+\alpha f(x, A) \mu\left(B_{\rho_{i} r_{k}}(x)\right) \\
\leq & \int_{B_{k} \backslash B_{k-1}} \alpha C\left(1+\left|A+P_{\mu}(x) \nabla v_{i, k}^{R}\right|^{p}\right) \mathrm{d} \mu^{x, \rho_{i}}+\int_{B_{R}} \alpha f\left(x, A+P_{\mu}(x) \nabla v_{i}^{R}\right) \mathrm{d} \mu^{x, \rho_{i}} .
\end{aligned}
$$

Taking a subsequence which runs well for $k=1, \ldots, K$, summing over $k$ and dividing by $K$, we find

$$
\begin{aligned}
& (1-\alpha) f(x, A) \mu\left(B_{\rho_{i}}(x)\right)+\alpha f(x, A) \frac{1}{K} \sum_{k=1}^{K} \mu\left(B_{\rho_{i} r_{k}}(x)\right) \\
\leq & \frac{1}{K} \int_{B_{R}} \alpha C\left(1+\left|A+P_{\mu}(x) \nabla v_{i, k}^{R}\right|^{p}\right) \mathrm{d} \mu^{x, \rho_{i}}+\int_{B_{R}} \alpha f\left(x, A+P_{\mu}(x) \nabla v_{i}^{R}\right) \mathrm{d} \mu^{x, \rho_{i}} .
\end{aligned}
$$

Dividing by $\mu\left(B_{\rho_{i}}(x)\right)$, and recalling that $r_{k} \geq r$, we obtain

$$
\begin{aligned}
(1-\alpha) f(x, A)+\alpha f(x, A) \frac{\mu\left(B_{\rho_{i} r}(x)\right)}{\mu\left(B_{\rho_{i}}(x)\right)} \leq & \frac{\alpha C 2^{p-1}}{K \mu\left(B_{\rho_{i}}(x)\right)} \int_{B_{R}}\left(1+|A|^{p}+\left|P_{\mu}(x) \nabla v_{i, k}^{R}\right|^{p}\right) \mathrm{d} \mu^{x, \rho_{i}} \\
& +\frac{\alpha}{\mu\left(B_{\rho_{i}}(x)\right)} \int_{B_{R}} f\left(x, A+P_{\mu}(x) \nabla v_{i}^{R}\right) \mathrm{d} \mu^{x, \rho_{i}} .
\end{aligned}
$$

Passing to the limsup as $i \rightarrow+\infty$, taking into account (5.11), we get, for some positive constant $\Lambda$ independent of $K$,

$$
(1-\alpha) f(x, A)+\alpha f(x, A) \nu\left(B_{r}\right) \leq \frac{\Lambda}{K}+\limsup _{i} \frac{\alpha}{\mu\left(B_{\rho_{i}}(x)\right)} \int_{B_{R}} f\left(x, A+P_{\mu}(x) \nabla v_{i}^{R}\right) \mathrm{d} \mu^{x, \rho_{i}}
$$

Letting $K \rightarrow+\infty, \alpha \rightarrow 1^{+}$, and $r \rightarrow R^{-}$, and taking into account that $\nu\left(B_{R}\right)=\lim _{i} \frac{\mu\left(B_{R \rho_{i}}(x)\right)}{\mu\left(B_{\rho_{i}}(x)\right)}$ by the choice of $R$, we finally get

$$
\begin{aligned}
f(x, A) & \leq \frac{1}{\nu\left(B_{R}\right)} \limsup _{i} \frac{1}{\mu\left(B_{\rho_{i}}(x)\right)} \int_{B_{R}} f\left(x, A+P_{\mu}(x) \nabla v_{i}^{R}\right) \mathrm{d} \mu^{x, \rho_{i}} \\
& =\limsup _{i} \frac{1}{\mu\left(B_{R \rho_{i}}(x)\right)} \int_{B_{R}} f\left(x, A+P_{\mu}(x) \nabla v_{i}^{R}\right) \mathrm{d} \mu^{x, \rho_{i}} \\
& =\limsup _{i} \frac{1}{\mu\left(B_{t_{i}}(x)\right)} \int_{B} f\left(x, A+P_{\mu}(x) \nabla v_{i}\right) \mathrm{d} \mu^{x, t_{i}},
\end{aligned}
$$

that is the required inequality (5.10).

During the preparation of this work, I had some stimulating discussions with Prof. L. Ambrosio, whom I wish to acknowledge. I also thank Prof. G. Bouchitté for his remarks in reading the paper.

\section{REFERENCES}

[1] E. Acerbi and N. Fusco, Semicontinuity problems in the Calculus of Variations. Arch. Rational Mech. Anal. 86 (1984) 125-145.

[2] L. Ambrosio, Introduzione alla Teoria Geometrica della Misura e Applicazioni alle Superfici Minime, Lectures Notes. Scuola Normale Superiore, Pisa (1996). 
[3] L. Ambrosio, On the lower-semicontinuity of quasi-convex integrals in SBV. Nonlinear Anal. 23 (1994) 405-425.

[4] L. Ambrosio, G. Buttazzo and I. Fonseca, Lower-semicontinuity problems in Sobolev spaces with respect to a measure. J. Math. Pures Appl. 75 (1996) 211-224.

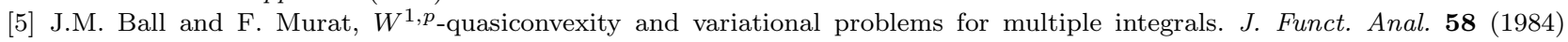
$225-253$.

[6] G. Bouchitté and G. Buttazzo, Characterization of optimal shapes and masses through Monge-Kantorovich equation. J. Eur. Math. Soc. 3 (2001) 139-168.

[7] G. Bouchitté, G. Buttazzo and I. Fragalà, Mean curvature of a measure and related variational problems. Ann. Scuola Norm. Sup. Pisa. Cl. Sci. IV XXV (1997) 179-196.

[8] G. Bouchitté, G. Buttazzo and I. Fragalà, Convergence of Sobolev spaces on varying manifolds. J. Geom. Anal. 11 (2001) 399-422.

[9] G. Bouchitté, G. Buttazzo and P. Seppecher, Energies with respect to a measure and applications to low dimensional structures. Calc. Var. Partial Differential Equations 5 (1997) 37-54.

[10] G. Bouchitté and I. Fragalà, Homogenization of thin structures by two-scale method with respect to measures. SIAM J. Math. Anal. 32 (2001) 1198-1126.

[11] G. Bouchitté and I. Fragalà, Homogenization of elastic thin structures: A measure-fattening approach. J. Convex. Anal. (to appear).

[12] A. Braides, Semicontinuity, Г-convergence and Homogenization for Multiple Integrals, Lectures Notes. SISSA, Trieste (1994).

[13] G. Buttazzo, Semicontinuity, Relaxation, and Integral Representation in the Calculus of Variations. Longman, Harlow, Pitman Res. Notes Math. Ser. 207 (1989).

[14] B. Dacorogna, Direct Methods in the Calculus of Variations. Springer-Verlag, Berlin, Appl. Math. Sci. 78 (1988).

[15] L.C. Evans and R.F. Gariepy, Measure Theory and Fine Properties of Functions. CRC Press, Ann Harbor, Stud. in Adv. Math. (1992).

[16] H. Federer, Geometric Measure Theory. Springer-Verlag, Berlin (1969).

[17] I. Fonseca and S. Müller, Quasi-convex integrands and lower semicontinuity in L'. SIAM J. Math. Anal. 23 (1992) 1081-1098.

[18] I. Fragalà and C. Mantegazza, On some notions of tangent space to a measure. Proc. Roy. Soc. Edinburgh 129A (1999) 331-342.

[19] P. Hajlasz and P. Koskela, Sobolev met Poincaré. Mem. Amer. Math. Soc. 145 (2000).

[20] P. Hajlasz and P. Koskela, Sobolev meets Poincaré. C. R. Acad. Sci. Paris 320 (1995) 1211-1215.

[21] A.D. Ioffe, On lower semicontinuity of integral functionals I and II. SIAM J. Contol Optim. 15 (1997) 521-538 and 991-1000.

[22] J. Kristensen, Lower semicontinuity in spaces of weakly differentiable functions. J. Math. Ann. 313 (1999) 653-710.

[23] J. Maly, Lower semicontinuity of quasiconvex integrals. Manuscripta Math. 85 (1994) 419-428.

[24] J.P. Mandallena, Contributions à une approche générale de la régularisation variationnelle de fonctionnelles intégrales, Thèse de Doctorat. Université de Montpellier II (1999).

[25] P. Marcellini, Approximation of quasiconvex functions, and lower semicontinuity of multiple integrals. Manuscripta Math. 51 (1985) 1-28.

[26] P. Marcellini and C. Sbordone, On the existence of minima of multiple integrals in the Calculus of Variations. J. Math. Pures Appl. 62 (1983) 1-9.

[27] P. Mattila, Geometry of Sets and Measures in Euclidean Spaces. Cambridge University Press, London (1995).

[28] C.B. Morrey, Multiple Integrals in the Calculus of Variations. Springer-Verlag, Berlin (1966).

[29] C. Olech, Weak lower semicontuity of integral functionals. J. Optim. Theory Appl. 19 (1976) 3-16.

[30] T. O'Neil, A measure with a large set of tangent measures. Proc. Amer. Math. Soc. 123 (1995) 2217-2221.

[31] D. Preiss, Geometry of measures on $\mathbb{R}^{n}$ : Distribution, rectifiability and densities. Ann. Math. 125 (1987) 573-643.

[32] L. Simon, Lectures on Geometric Measure Theory. Australian Nat. Univ., Proc. Centre for Math. Anal. 3 (1983).

[33] M. Valadier, Multiapplications mesurables à valeurs convexes compactes. J. Math. Pures Appl. 50 (1971) 265-297.

[34] V.V. Zhikov, On an extension and an application of the two-scale convergence method. Mat. Sb. 191 (2000) 31-72. 